\title{
On the largest component of subcritical random hyperbolic graphs*
}

\author{
Roland Diel $^{\dagger} \quad$ Dieter Mitsche ${ }^{\ddagger}$
}

\begin{abstract}
We consider the random hyperbolic graph model introduced by $\left[\mathrm{KPK}^{+} 10\right]$ and then formalized by [GPP12]. We show that, in the subcritical case $\alpha>1$, the size of the largest component is asymptotically almost surely $n^{1 /(2 \alpha)+o(1)}$, thus strengthening a result of [BFM15] which gave only an upper bound of $n^{1 / \alpha+o(1)}$.
\end{abstract}

Keywords: graph theory; geometric probability; random hyperbolic graphs. MSC2020 subject classifications: 05C80; 05C82; 60D05.

Submitted to ECP on March 2, 2020, final version accepted on February 5, 2021.

\section{Introduction and statement of result}

In the last decade, the model of random hyperbolic graphs introduced by Krioukov et al. in $\left[\mathrm{KPK}^{+} 10\right]$ was studied quite a bit due to its key properties also observed in large real-world networks. In [BnPK10] the authors showed empirically that the network of autonomous systems of the Internet can be very well embedded in the model of random hyperbolic graphs for a suitable choice of parameters. Moreover, Krioukov et al. [KPK $\left.{ }^{+} 10\right]$ gave empiric results that the model exhibits the algorithmic smallworld phenomenon established by the groundbreaking letter forwarding experiment of Milgram from the '60s [TM67]. From a theoretical point of view, the model of random hyperbolic graphs has an elegant specification and is thus amenable to rigorous analysis by mathematicians. Informally, the vertices are identified with points in the hyperbolic plane, and two vertices are connected by an edge if they are close in hyperbolic distance.

A common way of visualizing the hyperbolic plane is via its native representation described in $\left[\mathrm{BKL}^{+} 17 \mathrm{~b}\right]$ where the choice for ground space is $\mathbb{R}^{2}$. Here, a point of $\mathbb{R}^{2}$ with polar coordinates $(r, \theta)$ has hyperbolic distance to the origin $O$ equal to its Euclidean distance $r$ and more generally, the hyperbolic distance $\mathrm{d}\left(u, u^{\prime}\right)$ between two points $u=\left(r_{u}, \theta_{u}\right)$ and $u^{\prime}=\left(r_{u^{\prime}}, \theta_{u^{\prime}}\right)$ is obtained by solving

$$
\cosh \mathrm{d}\left(u, u^{\prime}\right):=\cosh r_{u} \cosh r_{u^{\prime}}-\sinh r_{u} \sinh r_{u^{\prime}} \cos \left(\theta_{u}-\theta_{u^{\prime}}\right) .
$$

In the native representation, an instance of the graph can be drawn by mapping a vertex $v$ to the point in $\mathbb{R}^{2}$ with polar coordinate $\left(r_{v}, \theta_{v}\right)$ and drawing edges as straight lines (see Figure 1).

\footnotetext{
${ }^{*}$ Roland Diel has been partially supported by grant GrHyDy ANR-20-CE40-0002. Dieter Mitsche has been partially supported by grant GrHyDy ANR-20-CE40-0002 and by IDEXLYON of Université de Lyon (Programme Investissements d'Avenir ANR16-IDEX-0005).

${ }^{\dagger}$ Université Côte d'Azur, CNRS, LJAD, France. E-mail: roland.diel@univ-cotedazur . fr.

${ }^{\ddagger}$ Institut Camille Jordan, Univ. Jean Monnet, Univ. St Etienne, Univ. Lyon, France. E-mail: dmitsche@ univ-st-etienne. fr.
} 


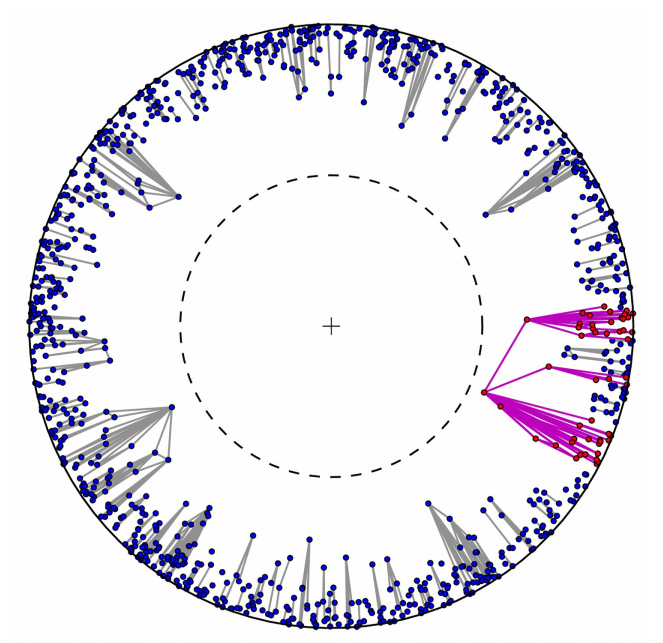

Figure 1: A realization of the subcritical hyperbolic graph $\operatorname{Poi}_{\alpha, \nu}(n)$ with parameters $\alpha=1.1, \nu=1, n=1000$. The outer circle of the figure corresponds to $B(O, R)$, the inner dashed circle is $B(O, R / 2)$. The size of the largest connected component, in purple, is $\left|L_{1}\right|=51$.

The random hyperbolic model is defined as follows: for each $n \in \mathbb{N}$, we consider a Poisson point process on the disk $B(O, R)$ of the hyperbolic plane. The radius is equal to $R:=2 \log (n / \nu)$ for some positive constant $\nu \in \mathbb{R}^{+}$(log denotes here and throughout the paper the natural logarithm). The intensity function at polar coordinates $(r, \theta)$ for $0 \leq r<R$ and $0 \leq \theta<2 \pi$ is

$$
g(r, \theta):=\nu e^{\frac{R}{2}} f(r, \theta),
$$

where $f(r, \theta)$ is the density function corresponding to the uniform probability on the disk $B(O, R)$ of the hyperbolic space of curvature $-\alpha^{2}$. That is, $\theta$ is chosen uniformly at random in the interval $[0,2 \pi)$ and independently of $r$, which is chosen according to the density function

$$
f(r):= \begin{cases}\frac{\alpha \sinh (\alpha r)}{\cosh (\alpha R)-1}, & \text { if } 0 \leq r<R \\ 0, & \text { otherwise. }\end{cases}
$$

Construct then the following graph $G=(V, E)$ : the set of vertices $V$ is the point set of the Poisson process and for $u, u^{\prime} \in V, u \neq u^{\prime}$, there is an edge with endpoints $u$ and $u^{\prime}$ provided the hyperbolic distance $\mathrm{d}\left(u, u^{\prime}\right)$ between $u$ and $u^{\prime}$ is such that $\mathrm{d}\left(u, u^{\prime}\right) \leq R$, where $\mathrm{d}\left(u, u^{\prime}\right)$ is obtained by solving (1.1).

For a given $n \in \mathbb{N}$, we denote this model by $\operatorname{Poi}_{\alpha, \nu}(n)$. Note in particular that

$$
\int g(r, \theta) d \theta d r=\nu e^{\frac{R}{2}}=n,
$$

and thus $\mathbb{E}|V|=n$. In the original model of Krioukov et al. [KPK $\left.{ }^{+} 10\right], n$ points, corresponding to vertices, are chosen uniformly and independently in the disk $B_{h}(O, R)$ of the hyperbolic space of curvature $-\alpha^{2}$, but since from a probabilistic point of view it is arguably more natural to consider the Poissonized version of this model, we consider the 
latter one (see also [GPP12] for the construction of the uniform model). Note also that conditional upon having exactly $n$ points in the Poisson process we recover exactly the uniform model.

The restriction $\alpha>\frac{1}{2}$ and the role of $R$ guarantee that the resulting graph has bounded average degree (depending on $\alpha$ and $\nu$ only). If $\alpha<\frac{1}{2}$, then the degree sequence is so heavy tailed that this is impossible (the graph is with high probability connected in this case, as shown in [BFM16]). Moreover, if $\alpha>1$, then as the number of vertices grows, the largest component of a random hyperbolic graph has sublinear order (see [BFM15, Theorem 1.4]).

Notation: We say that an event holds asymptotically almost surely (a.a.s.), if it holds with probability tending to 1 as $n \rightarrow \infty$. Given two sequences $\left(a_{n}\right)_{n \geq 1}$ and $\left(b_{n}\right)_{n \geq 1}$ taking values in $\mathbb{R}$ and such that $b_{n} \neq 0$ for $n$ large enough, we write $a_{n}=o\left(b_{n}\right)$ to mean that $\left|a_{n}\right| /\left|b_{n}\right| \rightarrow 0$ as $n \rightarrow \infty$. Also we write $a_{n}=\Theta\left(b_{n}\right)$ if $\left|a_{n}\right| /\left|b_{n}\right|$ is bounded away from 0 and $\infty$ as $n \rightarrow \infty$, and $a_{n}=\Omega\left(b_{n}\right)$ if $\left|a_{n}\right| /\left|b_{n}\right|$ is bounded away from 0 as $n \rightarrow \infty$.

Result: In this paper we study the size of the largest component of the graph in the case $\alpha>1$. In [BFM15, Theorem 1.4] it was shown that its size is a.a.s. at most $n^{1 / \alpha+o(1)}$. The main result of this paper is the following improvement, finding the exact exponent:

Theorem 1.1. Fix $\alpha>1$ and $\nu>0$ and let $G=(V, E)$ be chosen according to $\operatorname{Poi}_{\alpha, \nu}(n)$, and $L_{1} \subseteq G$ be the largest connected component of $G$. There is a constant $C>0$, such that, a.a.s., the following holds:

$$
n^{\frac{1}{2 \alpha}}(\log n)^{-C} \leq\left|L_{1}\right| \leq n^{\frac{1}{2 \alpha}}(\log n)^{C} .
$$

Remark 1.2. We cannot hope for Theorem 1.1 to hold for any $\alpha>1$ with probability at least $1-n^{-c}$ for some $c>0$. Indeed, the expected number of vertices $v$ with $r_{v} \leq$ $(1-\varepsilon)\left(1-\frac{1}{2 \alpha}\right) R$ (or equivalently $t_{v} \geq R\left(\frac{1-\varepsilon}{2 \alpha}+\varepsilon\right)$ ) is, by Lemma 2.3, $\Theta\left(n^{\varepsilon\left(\frac{1}{2 \alpha}-2 \alpha\right)}\right)=o(1)$, and hence the probability to have such a vertex is also of the same order. If such a vertex exists, by Lemma 2.5, its degree is (for some constant $c_{1}>0$ ) a.a.s. at least $c_{1} n^{(1-\varepsilon) /(2 \alpha)+\varepsilon}=n^{1 /(2 \alpha)+\varepsilon^{\prime}}$ for some $\varepsilon^{\prime}>0$. As before, the degree is a lower bound on the size of the component, and therefore we get with probability $n^{-c(\varepsilon)}$ (where $c(\varepsilon) \rightarrow 0$ as $\varepsilon \rightarrow 0$ ) a component of order at least $n^{1 /(2 \alpha)+\varepsilon^{\prime}}$ for some $\varepsilon^{\prime}>0$, and we cannot hope for stronger concentration.

Related work: The size of the largest component in random hyperbolic graphs was first studied in [BFM15]: it was shown that for $\alpha>1$ it is at most $n^{1 / \alpha+o(1)}$, whereas for $\alpha<1$ the largest component is linear. In the same paper the authors also showed that for $\alpha=1$ and $\nu$ sufficiently small there is a.a.s. no linear size component, whereas for $\alpha=1$ and $\nu$ sufficiently large a.a.s. there is a linear size component. In [FM18] the picture was made more precise: for $\alpha=1$ there is a critical intensity such that a.a.s. a linear size component exists iff $\nu$ is above a certain threshold. Also, for $\alpha<1$, for fixed $\alpha$, the size of the largest component is increasing in $\nu$ and, for fixed $\nu$, it is decreasing in $\alpha$. Furthermore, in [BFM16] it was shown that for $\alpha<1 / 2$ the graph is connected a.a.s., whereas for $\alpha=1 / 2$ the probability of being connected tends to 1 if $\nu \geq \pi$, and the probability of being connected is otherwise a monotone increasing function in $\nu$ that tends to 0 as $\nu$ tends to 0 . For the case $1 / 2<\alpha<1$, it was shown in [KM19] that a.a.s. the second component is of size $\Theta\left((\log n)^{1 /(1-\alpha)}\right)$, whereas, for $\alpha=1 / 2$ and $\nu$ sufficiently small, it is $\Theta(\log n)$ with constant probability, and for $\alpha=1$ it is a.a.s. $\Omega\left(n^{b}\right)$ for some $b>0$. More generally, starting with the seminal work of $\left[\mathrm{KPK}^{+} 10\right]$, further aspects of random hyperbolic graphs have been discussed since then: the power law degree distribution, mean degree and clustering coefficient were analyzed in [GPP12, FvdHMS21]; the diameter was computed in [FK18, KM15, MS19], the spectral gap was analyzed in [KM18], typical distances were calculated in [ABF17], and bootstrap percolation in such graphs was considered 
in [CF16]. The authors of [BFK16] calculated the size of balanced separators, i.e., small subsets of vertices whose removal yields subgraphs of roughly the same size, and they also compute the treewidth of random hyperbolic graphs. Finally, cliques in hyperbolic graphs were studied in [BFK18] and the vertex cover problem was studied in [BFFK20]. Recently, a more general model of geometric inhomogeneous random graphs has been introduced, see for example [BKL17a, BKL19, KL20] and the references therein.

Organization of the paper: In Section 2 we recall some well known properties of the random hyperbolic graph. Section 3 then describes the construction of the main tool of our proof: the separation zones. The existence of these zones shows that there is no long path of vertices with all vertices having roughly the same radial coordinates. Finally, in Section 4 we use the separation zones to control the size of the connected components of the graph which leads to the result of Theorem 1.1.

\section{Preliminaries}

From now on, we suppose $\alpha>1$. In this section we collect some properties concerning random hyperbolic graphs. For notational convenience, for any point $v=\left(r_{v}, \theta_{v}\right)$ of the ball $B(O, R)$ we define $t_{v}=R-r_{v}$, the radial distance to the boundary circle of radius $R$ (instead of the distance to the origin $O$ ), and we identify a vertex $v$ of the graph $G$ with the coordinate pair $v=\left(t_{v}, \theta_{v}\right)$ (this choice was made already in previous articles, see for example [CF16]). Moreover, the angles are defined modulo $2 \pi$, and the distance between two angles $\left|\theta-\theta^{\prime}\right|$ is a short hand notation for $\min \left(\left|\theta-\theta^{\prime}\right|, 2 \pi-\left|\theta-\theta^{\prime}\right|\right)$. For simplicity, we suppose throughout the paper that $R$ is an integer.

Define by $\theta_{\mathrm{d}}\left(\mathrm{d}_{1}, \mathrm{~d}_{2}\right)$ the angle at the origin between two points at radial distance $\mathrm{d}_{1}$ and $\mathrm{d}_{2}$ respectively from the origin, that are at hyperbolic distance $\mathrm{d}$ from each other (see Figure 2).

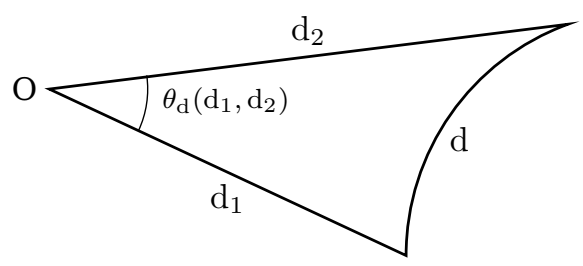

Figure 2: The angle $\theta_{\mathrm{d}}\left(\mathrm{d}_{1}, \mathrm{~d}_{2}\right)$

By the hyperbolic law of cosines of (1.1),

$$
\theta_{\mathrm{d}}\left(\mathrm{d}_{1}, \mathrm{~d}_{2}\right)=\arccos \left(\frac{\cosh \mathrm{d}_{1} \cosh \mathrm{d}_{2}-\cosh \mathrm{d}}{\sinh \mathrm{d}_{1} \sinh \mathrm{d}_{2}}\right) .
$$

Clearly, $\theta_{\mathrm{d}}\left(\mathrm{d}_{1}, \mathrm{~d}_{2}\right)=\theta_{\mathrm{d}}\left(\mathrm{d}_{2}, \mathrm{~d}_{1}\right)$. In fact, we need only a handy approximation for $\theta_{R}\left(\mathrm{~d}_{2}, \mathrm{~d}_{1}\right)$ :

Lemma 2.1 ([GPP12, Lemma 3.1]). If $0 \leq \min \left\{\mathrm{d}_{1}, \mathrm{~d}_{2}\right\} \leq R \leq \mathrm{d}_{1}+\mathrm{d}_{2}$, then

$$
\theta_{R}\left(\mathrm{~d}_{1}, \mathrm{~d}_{2}\right)=2 e^{\frac{1}{2}\left(R-\mathrm{d}_{1}-\mathrm{d}_{2}\right)}\left(1+\Theta\left(e^{R-\mathrm{d}_{1}-\mathrm{d}_{2}}\right)\right) .
$$

A direct consequence of this lemma is the following corollary:

Corollary 2.2. For any $R>0$, there is a function

$$
\theta^{R}: \begin{aligned}
{[0, R / 2]^{2} } & \rightarrow \mathbb{R}^{+} \\
\left(t_{1}, t_{2}\right) & \mapsto \theta^{R}\left(t_{1}, t_{2}\right)
\end{aligned}
$$

such that 
- $\theta^{R}\left(t_{1}, t_{2}\right)=2 e^{-\frac{1}{2}\left(R-t_{1}-t_{2}\right)}\left(1+\Theta\left(e^{-\left(R-t_{1}-t_{2}\right)}\right)\right)$

- two vertices $u, v \in V$ such that $t_{u}+t_{v} \leq R$ are connected by an edge iff $\left|\theta_{u}-\theta_{v}\right| \leq$ $\theta^{R}\left(t_{u}, t_{v}\right)$.

Throughout, we will need estimates for measures of regions of the hyperbolic plane, and more specifically, for regions obtained by performing some set algebra involving a few balls. For a point $p$ of the hyperbolic plane $\mathbb{H}^{2}$, the ball of radius $\rho$ centered at $p$ will be denoted by $B_{p}(\rho)$, i.e., $B_{p}(\rho):=\left\{q \in \mathbb{H}^{2}: \mathrm{d}(p, q) \leq \rho\right\}$. Also, we denote by $\mu(S)$ the measure of a set $S \subseteq \mathbb{H}^{2}$, i.e., $\mu(S):=\int_{S} f(r, \theta) d r d \theta$.

Next, we collect a few standard results for such measures.

Lemma 2.3 ([GPP12, Lemma 3.2]). Let $r \in(0, R]$ and denote $t=R-r$. Then

$$
\mu\left(B_{O}(r)\right)=\nu e^{-\alpha(R-r)}(1+o(1))=e^{-\alpha t}(1+o(1)) .
$$

We also use classical Chernoff concentration bounds for Poisson random variables. See for instance ([BLM13] page 23).

Lemma 2.4 (Chernoff bounds). If $X \sim \mathcal{P}(\lambda)$, then for any $x>0$,

$$
\mathbf{P}(X \geq \lambda+x) \leq e^{-\frac{x^{2}}{2(\lambda+x)}} \quad \text { and } \quad \mathbf{P}(X \leq \lambda-x) \leq e^{-\frac{x^{2}}{2(\lambda+x)}} .
$$

In particular, for $x \geq \lambda$,

$$
\mathbf{P}(X \geq 2 x) \leq e^{-\frac{x}{4}} .
$$

Lemma 2.4 together with Lemma 3.2 of [GPP12] yield the following result:

Lemma 2.5. Let $V$ be the vertex set of a graph chosen according to $\operatorname{Poi}_{\alpha, \nu}(n)$, and let $v$ be a vertex with $t_{v}>C \log R$ for $C$ sufficiently large. Then, there exist constants $0<c_{1}<c_{2}$, so that a.a.s. $c_{1} e^{\frac{1}{2} t_{v}} \leq\left|V \cap B_{v}(R)\right| \leq c_{2} e^{\frac{1}{2} t_{v}}$.

\section{Construction of the separation zones}

In this section we explain how to construct the separation zones. The high-level idea is as follows: in sectors of about the same angle we find separating regions without vertices at the angular boundary of the sector, whereas the zones in the middle of the center typically contain vertices. These separating regions on the angular borders of the sector therefore work as a natural boundary for the geometric region where the vertices of a connected component can be. In order for a connected component to span a very large angle, it would have to contain a vertex close to the origin, but since this region is typically also empty of vertices, no big connected component can exist. A similar separator concept was introduced in [BFK16]: the authors showed therein that for the given range of $\alpha>1$, there exist subsets of vertices of constant size such that in the remaining subgraph all components are roughly of the same size (the size of such separators given by the authors in [BFK16] is more interesting in the regime where there exists a large component, but their idea is similar to ours here). We explain the concepts now in more detail: we first define the following sectors

$$
S\left(\theta_{1}, \theta_{2}\right)=\left\{(t, \theta) \mid 0 \leq t<R \text { and } \theta_{1} \leq \theta<\theta_{2}\right\}
$$

and the annuli

$$
\mathcal{L}\left(t^{-}, t^{+}\right)=\left\{(t, \theta) \mid t^{-} \leq t<t^{+} \text {and } 0 \leq \theta<2 \pi\right\} .
$$

We first observe that the number of vertices contained in a not too narrow annulus of a ball centered at the origin is roughly of the same order as the total number of vertices in the whole ball. This is formalized in the following (which is a simple consequence of Lemma 2.3): 
Observation 3.1. For any $0 \leq t^{-}<t^{+}<R / 2$

$$
\mathbf{E}\left[\left|V \cap \mathcal{L}\left(t^{-}, t^{+}\right)\right|\right]=\nu e^{\frac{R}{2}-\alpha t^{-}}\left(1-e^{-\alpha\left(t^{+}-t^{-}\right)}+o(1)\right) .
$$

Proof. By applying Lemma 2.3 and the fact that the total intensity is $\nu e^{R / 2}$ we get

$$
\begin{aligned}
\mathbf{E}\left[\left|V \cap \mathcal{L}\left(t^{-}, t^{+}\right)\right|\right] & =\mathbf{E}\left[\left|V \cap B_{O}\left(t^{-}\right)\right| \backslash\left|V \cap B_{O}\left(t^{+}\right)\right|\right] \\
& =\nu e^{\frac{R}{2}-\alpha t^{-}}(1+o(1))-\nu e^{\frac{R}{2}-\alpha t^{+}}(1+o(1)) \\
& =\nu e^{\frac{R}{2}-\alpha t^{-}}\left(1-e^{-\alpha\left(t^{+}-t^{-}\right)}+o(1)\right) .
\end{aligned}
$$

We then construct for each coordinate pair $\left(t_{0}, \theta_{0}\right) \in(0, R / 2) \times[0,2 \pi)$, a zone that separates points to the left from points to the right in $\left\{(t, \theta), t \leq t_{0}\right\}$. More precisely, define for $t_{0}<R / 2$ and $\theta_{0} \in[0,2 \pi)$, the following separation zone (see also Figure 3 ):

$$
\mathcal{A}\left(t_{0}, \theta_{0}\right)=\left\{(t, \theta) \mid t \leq t_{0} \text { and }\left|\theta-\theta_{0}\right| \leq \theta^{R}(t, t)\right\} .
$$

We thus have the following observation (angles in the following observation are chosen to be the smaller angle with respect to the given reference angle $\theta_{0}$ ):

Observation 3.2. Suppose $V \cap \mathcal{A}\left(t_{0}, \theta_{0}\right)=\emptyset$. Let $v, w \in V \cap \mathcal{L}\left(0, t_{0}\right)$ with $\theta_{v}<\theta_{0}<\theta_{w}$ and $\max \left(\left|\theta_{v}-\theta_{0}\right|,\left|\theta_{w}-\theta_{0}\right|\right) \leq\left|\theta_{v}-\theta_{w}\right|$. Then $\left|\theta_{v}-\theta_{w}\right|>\theta^{R}\left(t_{v}, t_{w}\right)$, i.e., $v$ and $w$ are not connected by an edge.

Proof. The function $\theta^{R}\left(t_{v}, t_{w}\right)$ is increasing in both of its arguments, hence we may assume that $t_{v}=t_{w}=t_{0}$. For this choice, $v$ and $w$ are connected by Corollary 2.2 iff $\left|\theta_{v}-\theta_{w}\right| \leq \theta^{R}\left(t_{0}, t_{0}\right)$. However, since $\mathcal{A}\left(t_{0}, \theta_{0}\right)=\emptyset$ and $\theta_{v}<\theta_{0}<\theta_{w}$, we have $\left|\theta_{v}-\theta_{w}\right|>2 \theta^{R}\left(t_{0}, t_{0}\right)$, i.e., $v$ and $w$ are not connected by an edge.

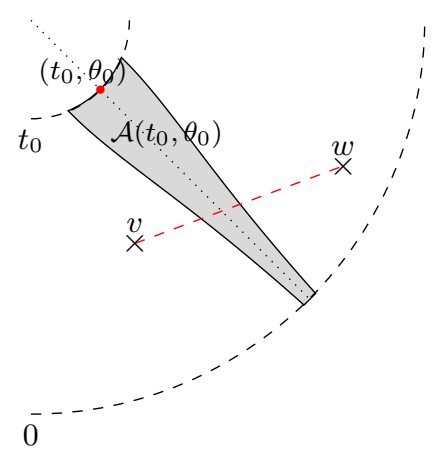

Figure 3: A separation zone. The two points $v$ and $w$ are not close enough to be connected by an edge.

To use the previous observation, we need separation zones which do not contain any vertices. We prove below that this happens with high probability.

Lemma 3.3. There is a constant $c>0$ which depends only on $\alpha$ and $\nu$ such that for any $t<R / 2$,

$$
\mathbf{P}\left(\exists j \in\{0, \ldots, c R\}, V \cap \mathcal{A}\left(t, 2 j \theta^{R}(t, t)\right)=\emptyset\right) \geq 1-e^{-R} .
$$

Proof. Consider the event

$$
E=\left\{\exists j \in\{0, \ldots, N\}, V \cap \mathcal{A}\left(t, 2 j \theta^{R}(t, t)\right)=\emptyset\right\}
$$


for some $N$ that we will choose below. We recall that the set $\mathcal{A}\left(t, 2 j \theta^{R}(t, t)\right)$ is included in the sector

$$
S\left((2 j-1) \theta^{R}(t, t),(2 j+1) \theta^{R}(t, t)\right) .
$$

For different values of $j$, these sectors are disjoint, and thus the random variables $\left|V \cap \mathcal{A}\left(t, 2 j \theta^{R}(t, t)\right)\right|$ are independent, they follow a Poisson distribution with intensity $\mathbf{E}[|V \cap \mathcal{A}(t, 0)|]$, and hence

$$
\mathbf{P}(\bar{E})=(\mathbf{P}(|V \cap \mathcal{A}(t, 0)|>0))^{N+1}=\left(1-e^{-\mathbf{E}[|V \cap \mathcal{A}(t, 0)|]}\right)^{N+1} .
$$

Since $t<R / 2$, Corollary 2.2 and Observation 3.1 give

$$
\begin{aligned}
\mathbf{E}[|V \cap \mathcal{A}(t, 0)|] & \leq \sum_{1 \leq i \leq\lceil t\rceil} \frac{2 \theta^{R}(i, i)}{2 \pi} \mathbf{E}[|V \cap \mathcal{L}(i-1, i)|] \\
& \leq \sum_{1 \leq i \leq\lceil t\rceil} 4 e^{-\frac{R}{2}+i} \nu e^{R / 2} e^{-\alpha(i-1)}\left(1-e^{-\alpha}\right)(1+o(1)) \\
& =4 \nu\left(e^{\alpha}-1\right) \sum_{1 \leq i \leq\lceil t\rceil} e^{-(\alpha-1) i}(1+o(1)) \\
& \leq 4 \nu \frac{\left(e^{\alpha}-1\right) e^{-(\alpha-1)}}{1-e^{-(\alpha-1)}}+o(1) .
\end{aligned}
$$

By choosing $N=c R$ for a constant $c>0$ sufficiently large, the lemma follows (observe that the angle $\theta^{R}(i, i)$ is small enough to have $2 N \theta^{R}(i, i)<2 \pi$ for large $R$ and then such $N$ clearly exists).

We next introduce a helpful structure of layers that had in a similar way been introduced in [BKL17a] for an efficient sampling of geometric inhomogeneous random graphs. In more detail, we consider the following layers starting from the boundary of $B_{O}(R)$ : set

$$
\forall i \geq 0, t_{i}=\left(\frac{4 \alpha}{\alpha-1}+3 i\right) \log R .
$$

Let $t_{\max }=\frac{1}{2 \alpha} R$ be the distance to the circle of radius $R$ roughly corresponding to the largest $t$ for which we can find an element of $V$ and set $i_{\max }=\min \left\{i \geq 0, t_{i} \geq t_{\max }\right\}$. Note that

$$
t_{\max }<R / 2, i_{\max } \leq R \text { and } t_{\max } \leq t_{i_{\max }} \leq t_{\max }+3 \log R .
$$

We also set $t_{-1}=0$ and we define, for $i, j \in\left\{0, \ldots, i_{\max }\right\}$, the angle

$$
\theta_{i, j}=\theta^{R}\left(t_{i}, t_{j}\right)
$$

and the consecutive layers

$$
\mathcal{L}_{i}=\mathcal{L}\left(t_{i-1}, t_{i}\right) .
$$

The following observation is standard; it follows again from Corollary 2.2 and Observation 3.1. Its proof is analogous to the proof of Observation 3.1; and it is therefore left out.

Observation 3.4. For any $i, j \in\left\{0, \ldots, i_{\max }\right\}$,

$$
\mathbf{E}\left[\left|V \cap \mathcal{L}_{i}\right|\right]=\nu e^{\frac{R}{2}-\alpha t_{i-1}}(1+o(1)) \quad \text { and } \quad \theta_{i, j}=2 e^{-\frac{1}{2}\left(R-t_{i}-t_{j}\right)}\left(1+\Theta\left(e^{-\left(R-t_{i}-t_{j}\right)}\right)\right) .
$$

We now finish the construction of the separation zones. Intuitively, one may think of separation zones as regions equally distributed over $B_{O}(R)$, but it seems easier to define these regions depending on positions of vertices. On a high level, the idea is 
as follows: we first divide the disk into layers (concentric rings). Each layer is then divided into sectors by packing separation zones as close to each other as possible the number of zones depends on the layer). More formally, for every $i \in\left\{0, \ldots, i_{\max }\right\}$, set $k_{\max }^{i}=\left\lceil 2 \pi /\left(3 c R \theta_{i, i}\right)\right\rceil$ where $c$ is the constant given in Lemma 3.3. For every $0 \leq k<k_{\max }^{i}$, we find the $(k+1)$-th separation zone to be the closest (to the right) empty region to the angle $3 c R k \theta_{i, i}$. More formally, define for $0 \leq k<k_{\max }^{i}$,

$$
j^{i, k}=\min \left\{j \in \mathbb{N} \mid V \cap \mathcal{A}\left(t_{i},(3 c R k+2 j) \theta_{i, i}\right)=\emptyset\right\},
$$

where $\min \emptyset=\infty$. We assign $\mathcal{A}^{i, k}$ then to be the closest region to $3 c R k \theta_{i, i}$ :

$$
\mathcal{A}^{i, k}=\mathcal{A}\left(t_{i},\left(3 c R k+2 j^{i, k}\right) \theta_{i, i}\right),
$$

where we set $\mathcal{A}^{i, k}=\emptyset$ in case it turned out $j^{i, k}=\infty$. The set $\mathcal{A}^{i, k}$ represents the $(k+1)$-th separation zone of layer $i$. For notational convenience, we also set $\mathcal{A}^{i, k_{\max }^{i}}=\mathcal{A}^{i, 0}$. We could have $\mathcal{A}^{i, k}=\mathcal{A}^{i, k+1}$, and the two sets might not even be well defined. We will thus use Lemma 3.3 to show that asymptotically almost surely none of the two things happens.

In order to state the next lemma properly, we define the following (pseudo)distance between separation zones:

$$
\forall A, B \subset B_{O}(R), d(A, B)=\inf \left\{\left|\theta-\theta^{\prime}\right| \mid(t, \theta) \in A,\left(t^{\prime}, \theta^{\prime}\right) \in B\right\} .
$$

As indicated before, the separation zones described indeed are well defined, i.e., they are more or less equally distributed around the whole disk, as the following lemma shows:

Lemma 3.5. Let $c$ be the constant given in Lemma 3.3 (depending only on $\alpha$ and $\nu$ ). Then the event $\mathcal{E}_{R}$ defined by

$$
\mathcal{E}_{R}=\left\{\forall 0 \leq i \leq i_{\max }, \forall 0 \leq k<k_{\max }^{i}, \mathcal{A}^{i, k} \neq \emptyset \text { and } c R \theta_{i, i} \leq d\left(\mathcal{A}^{i, k}, \mathcal{A}^{i, k+1}\right) \leq 5 c R \theta_{i, i}\right\}
$$

occurs a.a.s.

Proof. Let $c$ be the constant given in Lemma 3.3 and consider the event

$$
\mathcal{F}_{R}=\left\{\forall 0 \leq i \leq i_{\text {max }}, \forall 0 \leq k<k_{\text {max }}^{i}, \exists j \in\{0, \ldots, c R\}, V \cap \mathcal{A}\left(t_{i},(3 c R k+2 j) \theta_{i, i}\right)=\emptyset\right\} .
$$

Clearly, $\mathcal{F}_{R} \subseteq \mathcal{E}_{R}$ and it is sufficient to bound $\mathbf{P}\left(\overline{\mathcal{F}_{R}}\right)$. Then, for $R$ large enough, using the definition of $k_{\max }^{i}$,

$$
\begin{aligned}
\mathbf{P}\left(\overline{\mathcal{F}_{R}}\right) & \leq \sum_{\substack{0 \leq i \leq i_{\max } \\
0 \leq k<k_{\max }^{i}}} \mathbf{P}\left(\forall j \in\{0, \ldots, c R\}, V \cap \mathcal{A}\left(t_{i},(3 c R k+2 j) \theta_{i, i}\right) \neq \emptyset\right) \\
& =\sum_{0 \leq i \leq i_{\max }} k_{\max }^{i} \mathbf{P}\left(\forall j \in\{0, \ldots, c R\}, V \cap \mathcal{A}\left(t_{i}, 2 j \theta_{i, i}\right) \neq \emptyset\right) \\
& =C_{1} \sum_{0 \leq i \leq i_{\max }} \frac{1}{R} e^{\frac{R}{2}-t_{i}} e^{-R} \\
& \leq C_{1} \sum_{0 \leq i \leq i_{\max }} e^{\frac{R}{2}-t_{i}} e^{-R} \leq C_{2} e^{-\frac{1}{2} R} .
\end{aligned}
$$

Since the last quantity goes to zero as $R$ tends to infinity, the lemma is proven.

Hence, a.a.s. the distance between two consecutive separation zones $\mathcal{A}^{i, k}$ and $\mathcal{A}^{i, k+1}$ is always of the order $R \theta_{i, i}$. The idea is that every path of connected points from a vertex in $V \cap \mathcal{B}_{i, k}$ to another vertex in $V \cap \mathcal{B}_{i, \ell}$ with $k \neq \ell$ has to go through an intermediate 


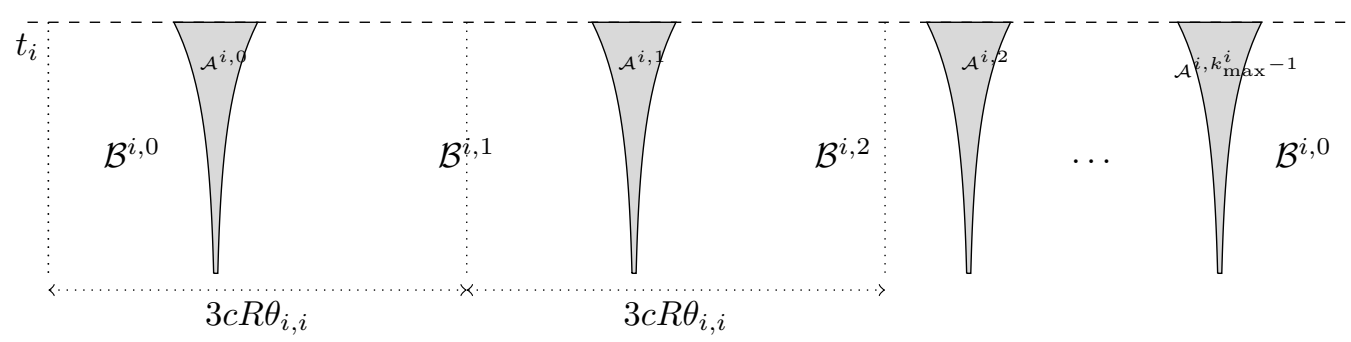

Figure 4: The separation zones

vertex that is closer to the origin, i.e., points cannot be connected "below". In order to make this more precise, define now, conditional upon $\mathcal{E}_{R}$, the area $\mathcal{B}_{i, k}$ between two separation zones (see also Figure 4): for $1 \leq k<k_{\max }^{i}$, let

$$
\begin{aligned}
& \mathcal{B}_{i, k}=\left\{(t, \theta) \in B_{O}(R) \mid t \leq t_{i}\right. \\
& \left.\quad \text { and } \sup \left\{\tilde{\theta} \mid(t, \tilde{\theta}) \in \mathcal{A}^{i, k-1}\right\}<\theta<\inf \left\{\tilde{\theta} \mid(t, \tilde{\theta}) \in \mathcal{A}^{i, k}\right\}\right\} \\
& \text { and } \mathcal{B}_{i, 0}=\left\{(t, \theta) \in B_{O}(R) \mid t \leq t_{i}\right. \\
& \left.\quad \text { and } \sup \left\{\tilde{\theta} \mid(t, \tilde{\theta}) \in \mathcal{A}^{i, k_{\max }^{i}-1}\right\}<\theta \text { or } \theta<\inf \left\{\tilde{\theta} \mid(t, \tilde{\theta}) \in \mathcal{A}^{i, 0}\right\}\right\} .
\end{aligned}
$$

Rewriting Observation 3.2 we obtain the following observation, assuming that we indeed found the separation zones, that is, we have $\mathcal{A}^{i, k} \neq \emptyset$.

Observation 3.6. Suppose $\mathcal{A}^{i, k} \neq \emptyset$. Let $u \in V \cap \mathcal{B}_{i, k}$ and $v \in V \cap \mathcal{B}_{i, \ell}$ with $k \neq \ell$. Then $u$ and $v$ can only be connected by a path that has at least one intermediate vertex $w \in V$ such that $t_{w}>t_{i}$.

Proof. Note that $t_{u}, t_{v} \leq t_{i}$. Then by Observation 3.2, $u$ and $v$ are not connected by an edge. Since this holds for any $k \neq \ell$ and any $u, v$, there can be no path between $u$ and $v$ containing vertices $w \in V$ such that $t_{w} \leq t_{i}$ (see also Figure 5).

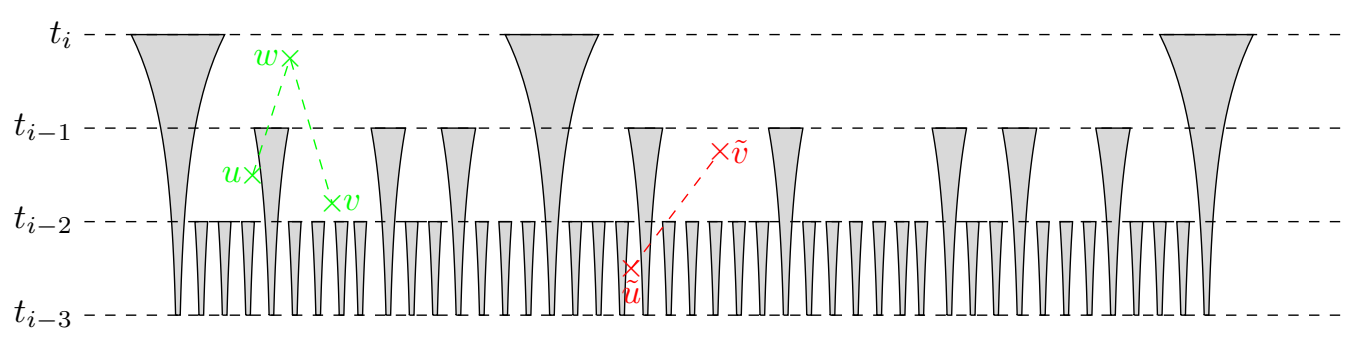

Figure 5: The green points are connected while the red ones are not.

\section{Covering component}

On a high level, the advantage of separation zones is that it is impossible to stay in the same connected component going from right to left (or the other direction) remaining always at the same radius or going towards the boundary. We will thus construct, starting from a certain vertex, a covering component, that is, a component which covers a.a.s. 
the whole connected component of the vertex, if this vertex is the one closest to the center of its connected component. Roughly speaking, it is constructed in an iterative way and contains vertices that are 'close' in angular distance to a vertex already in the covering component, where 'close' depends on the radial coordinate of the vertex.

We describe now in detail the iterative construction process of the covering component. Suppose that the event $\mathcal{E}_{R}$ holds. This happens a.a.s. according to Lemma 3.5. Consider a vertex $v \in V$ and let $\mathcal{L}_{i}$ be the layer of $v$. If $i=0$, we define $C_{v}=\{v\}$, else, for $0 \leq j<i \leq i_{\max }$, we set

$$
\Theta_{i, j}(v)=V \cap \mathcal{L}_{j} \cap S\left(\theta_{v}-2 \theta_{i, j}, \theta_{v}+2 \theta_{i, j}\right)
$$

and (see also Figure 6)

$$
C_{v}=\{v\} \cup \bigcup_{j=0}^{i-1} \bigcup_{u \in \Theta_{i, j}(v)} C_{u}
$$

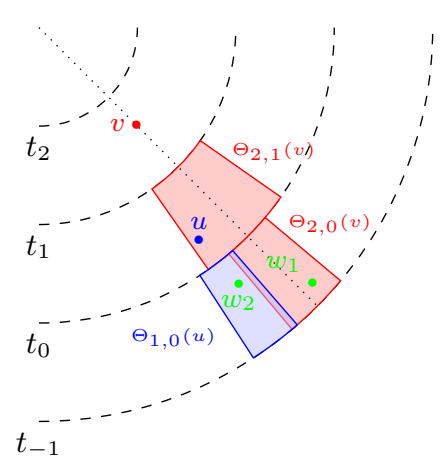

Figure 6: Construction of $C_{v}=\left\{v, u, w_{1}, w_{2}\right\}$, for $v \in \mathcal{L}_{2}$ top-down: first $u$ is added since this vertex lies in $\Theta_{2,1}(v)$, in red, then $w_{1}$ since it is in $\Theta_{2,0}(v)$, in red too, and finally $w_{2}$ is added since it lies in $\Theta_{1,0}(u)$, in blue.

Finally, denote by $k$ the unique integer such that $v \in \mathcal{L}_{i} \cap \mathcal{B}_{i, k}$. The covering component of $v$ is defined as

$$
\mathbf{C C}_{v}=\bigcup_{u \in V \cap \mathcal{L}_{i} \cap \mathcal{B}_{i, k}} C_{u} .
$$

We also denote by $\operatorname{Conn}(v)$ the connected component of $v$. The following lemma shows that the covering component of $v$ indeed covers the connected component of $v$ if $v$ is the closest vertex of the center in this component.

Lemma 4.1. A.a.s. for any $v \in B_{O}(R)$, if $t_{v}=\max \left\{t_{u} \mid u \in \operatorname{Conn}(v)\right\}$, the connected component of $v$ is included in $\mathbf{C C}_{v}$.

Proof. Suppose that the event $\mathcal{E}_{R}$ holds. This happens a.a.s. according to Lemma 3.5.

By contradiction, consider a vertex $u$ in the connected component of $v$ that is not contained in $\mathbf{C C}_{v}$, and a shortest path $v=v_{0}, \ldots, v_{m}=u$. Hence, there exists a smallest $k \geq 1$ such that the vertex $v_{k}$ is not in $\mathbf{C C}_{v}$.

For any $\ell \in\{0, \ldots, m\}$, denote by $i_{\ell}$ the index of the layer containing $v_{\ell}$ and suppose there exists $k^{\prime}<k$ such that $i_{k^{\prime}}>i_{k}$. Among them choose the largest $k^{\prime}$, so that

$$
\forall \ell \in\left\{k^{\prime}+1, \ldots, k\right\}, i_{\ell} \leq i_{k} \quad \text { (see Figure 7) }
$$




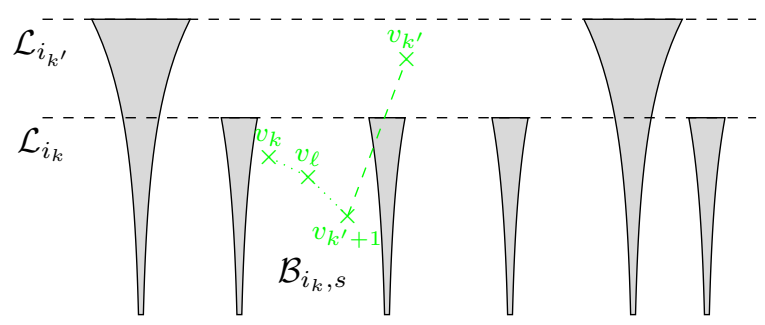

Figure 7: The whole path after $v_{k^{\prime}}$ is contained in a unique zone $\mathcal{B}_{i_{k}, s}$.

By Observation 3.6, it is not possible to construct a path between vertices of different zones of level $i_{k}$ if this path does not contain a vertex $w$ such that $t_{w}>t_{i_{k}}$. Hence, the $v_{\ell}$ are necessarily in the same zone as $v_{k}$, named $\mathcal{B}_{i_{k}, s}$ in Figure 7 . Therefore, the angle between $v_{k^{\prime}}$ and $v_{k}$ cannot be larger than the angle between $v_{k^{\prime}}$ and $v_{k^{\prime}+1}$ plus the angular width of $\mathcal{B}_{i_{k}, s}$, that is,

$$
\left|\theta_{v_{k^{\prime}}}-\theta_{v_{k}}\right| \leq \theta_{i_{k^{\prime}} i_{k}}+5 c R \theta_{i_{k} i_{k}} \leq 2 \theta_{i_{k^{\prime}} i_{k}}
$$

and thus $v_{k} \in C_{v_{k^{\prime}}} \subset \mathbf{C C}_{v}$ which is impossible. Thus necessarily $v=v_{0}$ is in the same layer as $v_{k}$ or in a layer closer to the boundary. Since $v$ is by hypothesis the vertex such that $t_{v}=\max \left\{t_{u} \mid u \in \operatorname{Conn}(v)\right\}$, we must have $v \in \mathcal{L}_{i_{k}}$. Therefore, by Observation 3.6, $v$ and $v_{k}$ must be in the same zone $\mathcal{B}_{i_{k}, s}$ for some $s$ and thus $v_{k} \in \mathbf{C C}_{v}$.

Lemma 4.2. Define $K=128 \nu$. A.a.s.,

$$
\forall 0 \leq j<i \leq i_{\max }, \forall v \in \mathcal{L}_{i},\left|\Theta_{i, j}(v)\right| \leq \max \left(8 R, K e^{\frac{t_{i}+t_{j}}{2}-\alpha t_{j-1}}\right)
$$

Proof. For each $0 \leq j<i \leq i_{\max }$, let $d_{i, j}=\max \left(4 R, 64 \nu e^{\frac{t_{i}+t_{j}}{2}-\alpha t_{j-1}}\right)$ and divide layer $\mathcal{L}_{j}$ into $\left\lceil\pi /\left(2 \theta_{i, j}\right)\right\rceil$ sectors $S_{k}^{(i, j)}$ of angle (at most) $4 \theta_{i, j}$. Recall that for any $k \in\left\{1, \ldots,\left\lceil\pi /\left(2 \theta_{i, j}\right)\right\rceil\right\}$, the cardinality $\left|S_{k}^{(i, j)} \cap V \cap \mathcal{L}_{j}\right|$ is Poisson distributed with expectation

$$
\mathbf{E}\left[\left|S_{k}^{(i, j)} \cap V \cap \mathcal{L}_{j}\right|\right] \leq 4 \theta_{i, j} \mathbf{E}\left[\left|V \cap \mathcal{L}_{j}\right|\right]
$$

and according to Observation 3.4, if $R$ is large enough,

$$
4 \theta_{i, j} \mathbf{E}\left[\left|V \cap \mathcal{L}_{j}\right|\right] \leq 4 \nu e^{\frac{R}{2}-\alpha t_{j-1}} \cdot 2 e^{-\frac{1}{2}\left(R-t_{i}-t_{j}\right)}=8 \nu e^{\frac{t_{i}+t_{j}}{2}-\alpha t_{j-1}} \leq d_{i, j} / 2 .
$$

So, for any such sector $S_{k}^{(i, j)}$, for any $0 \leq j<i \leq i_{\max }$, from Lemma 2.4 we have

$$
\mathbf{P}\left(\left|S_{k}^{(i, j)} \cap V \cap \mathcal{L}_{j}\right|>d_{i, j}\right) \leq e^{-d_{i, j} / 4} \leq e^{-R} .
$$

By a union bound over all $\left\lceil\pi /\left(2 \theta_{i, j}\right)\right\rceil$ sectors $S_{k}^{(i, j)}$ and then over all $i, j$, we have

$$
\begin{aligned}
& \mathbf{P}\left(\exists 0 \leq j<i \leq i_{\max }, \exists 1 \leq k \leq\left\lceil\pi /\left(2 \theta_{i, j}\right)\right\rceil,\left|S_{k}^{(i, j)} \cap V \cap \mathcal{L}_{j}\right|>d_{i, j}\right) \\
\leq & \sum_{0 \leq j<i \leq i_{\max }}\left\lceil\pi /\left(2 \theta_{i, j}\right)\right\rceil e^{-R} .
\end{aligned}
$$

Recall that for $R$ large enough, $1 / \theta_{i, j} \leq e^{\frac{1}{2}\left(R-t_{i}-t_{j}\right)} \leq e^{R / 2}$ and $i_{\max } \leq R$. Thus, for some universal constant $C>0$,

$$
\mathbf{P}\left(\exists 0 \leq j<i \leq i_{\max }, \exists 1 \leq k \leq\left\lceil\pi /\left(2 \theta_{i, j}\right)\right\rceil,\left|S_{k}^{(i, j)} \cap V \cap \mathcal{L}_{j}\right|>d_{i, j}\right) \leq C R^{2} e^{-R / 2}=o(1) .
$$


Hence, since for each vertex $v \in V \cap \mathcal{L}_{i}$, the set $\Theta_{i, j}(v)$ can intersect at most two adjacent sectors $S_{k}^{(i, j)}$, we have

$$
\mathbf{P}\left(\exists 0 \leq j<i \leq i_{\max }, \exists v \in V \cap \mathcal{L}_{i},\left|\Theta_{i, j}(v)\right|>2 d_{i, j}\right)=o(1) .
$$

Lemma 4.3. There is a constant $K_{2}>0$, such that, a.a.s.

$$
\forall 0 \leq i \leq i_{\max }, \forall v \in \mathcal{L}_{i},\left|\mathbf{C C}_{v}\right| \leq K_{2} e^{2 t_{0}+\frac{1}{2} t_{i}} .
$$

Proof. Upper bound for $\left|C_{v}\right|$ : recall first that Lemma 4.2 says that, for $K=128 \nu$, the event

$$
\mathcal{A}=\left\{\forall 0 \leq j<i \leq i_{\max }, \forall v \in V \cap \mathcal{L}_{i},\left|\Theta_{i, j}(v)\right| \leq \max \left(8 R, K e^{\frac{t_{i}+t_{j}}{2}-\alpha t_{j-1}}\right)\right\}
$$

happens a.a.s. We proceed by induction on $i$ and prove that, on the event $\mathcal{A}$, for any $0 \leq i \leq i_{\max }$

$$
\forall j \leq i, \forall v \in V \cap \mathcal{L}_{j},\left|C_{v}\right| \leq 2 K e^{\frac{t_{0}+t_{i}}{2}} .
$$

Since for any $v \in V \cap \mathcal{L}_{0}, C_{v}=\{v\}$, the result is obvious for $i=0$. Suppose now it is true for some $0 \leq i<i_{\max }$. We fix some $v \in V \cap \mathcal{L}_{i+1}$, and we will obtain a bound on $C_{v}$ by summing over all vertices in layers with indices below $i+1$ and take their $C_{u}$ 's:

$$
\begin{aligned}
\left|C_{v}\right| & \leq|\{v\}|+\sum_{0 \leq j \leq i} \sum_{u \in \Theta_{i+1, j}(v)}\left|C_{u}\right| \\
& \leq 1+\sum_{u \in \Theta_{i+1,0}(v)} 1+\sum_{1 \leq j \leq i} \sum_{u \in \Theta_{i+1, j}(v)} 2 K e^{\frac{t_{0}+t_{i}}{2}} \\
& =1+\left|\Theta_{i+1,0}(v)\right|+\sum_{1 \leq j \leq i}\left|\Theta_{i+1, j}(v)\right| 2 K e^{\frac{t_{0}+t_{i}}{2}} \\
& \leq 1+\max \left(8 R, K e^{\frac{t_{i+1}+t_{0}}{2}-\alpha t_{-1}}\right)+\sum_{1 \leq j \leq i} \max \left(8 R, K e^{\frac{t_{i+1}+t_{j}}{2}-\alpha t_{j-1}}\right) 2 K e^{\frac{t_{0}+t_{j}}{2}} .
\end{aligned}
$$

Recall that $t_{-1}=0$ and for $i \geq 0, t_{i}=\left(\frac{4 \alpha}{\alpha-1}+3 i\right) \log R$. Thus, for large $R$,

$$
\max \left(8 R, K e^{\frac{t_{i+1}+t_{0}}{2}-\alpha t_{-1}}\right)=K e^{\frac{t_{i+1}+t_{0}}{2}}
$$

and

$$
\begin{aligned}
\max \left(8 R, K e^{\frac{t_{i+1}+t_{j}}{2}-\alpha t_{j-1}}\right) 2 K e^{\frac{t_{0}+t_{j}}{2}} & \leq 2 K e^{\frac{t_{0}+t_{i+1}}{2}}\left(8 R e^{\frac{t_{j}-t_{i+1}}{2}}+K e^{t_{j}-\alpha t_{j-1}}\right) \\
& =2 K e^{\frac{t_{0}+t_{i+1}}{2}}\left(8 R^{\frac{3}{2}(j-i)-\frac{1}{2}}+K R^{-\alpha-3(\alpha-1) j}\right) .
\end{aligned}
$$

This leads to the following bound for $\left|C_{v}\right|$ :

$$
\left|C_{v}\right| \leq 1+e^{\frac{t_{0}+t_{i+1}}{2}}\left(K+\frac{16 K}{\sqrt{R}\left(1-R^{-3 / 2}\right)}+2 K^{2} \frac{R^{-4 \alpha+3}}{1-R^{-3(\alpha-1)}}\right)
$$

For $R$ large enough, we then have $\left|C_{v}\right| \leq 2 K e^{\frac{t_{0}+t_{i+1}}{2}}$ for any $v \in \mathcal{L}_{i+1}$. This proves the bound of (4.1) for any $v$ by induction.

Upper bound for $\left|\mathbf{C C}_{v}\right|:$ For $i \in\left\{0, \ldots, i_{\max }\right\}$, denote by $\Gamma_{i}$ the set

$$
\Gamma_{i}=\left\{v \in V \cap \mathcal{L}_{i}|| \theta_{v} \mid \leq 5 c R \theta_{i, i}\right\} .
$$


According to Observation 3.4, there is a constant $K$ depending only on $\nu$ and $c$ such that for $R$ large enough and $i \in\left\{0, \ldots, i_{\max }\right\}$,

$$
\mathbf{E}\left[\left|\Gamma_{i}\right|\right]=10 c R \theta_{i, i} \mathbf{E}\left[\left|V \cap \mathcal{L}_{i}\right|\right] \leq K R e^{-\frac{1}{2}\left(R-2 t_{i}\right)} e^{\frac{R}{2}-\alpha t_{i-1}}=K R e^{t_{i}-\alpha t_{i-1}}
$$

Thus, $\mathbf{E}\left[\left|\Gamma_{i}\right|\right] \leq K R e^{t_{0}} \leq \frac{1}{2} e^{\frac{3}{2} t_{0}}$. Now, since $\left|\Gamma_{i}\right|$ is a Poisson variable, Lemma 2.4 says that

$$
\mathbf{P}\left(\left|\Gamma_{i}\right| \geq e^{\frac{3}{2} t_{0}}\right) \leq e^{-e^{\frac{3}{2} t_{0}} / 8}
$$

Therefore,

$$
\begin{aligned}
& \mathbf{P}\left(\mathcal{E}_{R} \cap\left\{\exists i \in\left\{0, \ldots, i_{\max }\right\}, \exists k \in\left\{1, \ldots, k_{\max }^{i}\right\},\left|V \cap \mathcal{L}_{i} \cap \mathcal{B}_{i, k}\right| \geq e^{\frac{3}{2} t_{0}}\right\}\right) \\
& \leq \sum_{i=0}^{i_{\max }}\left[\frac{1}{2 c R \theta_{i, i}}\right] \mathbf{P}\left(\left|\Gamma_{i}\right| \geq e^{\frac{3}{2} t_{0}}\right) \\
& \leq \sum_{i=0}^{i_{\max }} \frac{1}{c R} e^{\frac{R}{2}-t_{i}} e^{-e^{\frac{3}{2} t_{0}} / 8} \leq e^{R / 2-e^{\frac{3}{2} t_{0}} / 8}
\end{aligned}
$$

which tends to 0 as $R$ goes to infinity.

Finally, a.a.s., for any $i \geq 0$ and any $v \in V \cap \mathcal{L}_{i}$, the cardinality of $\mathbf{C C}_{v}$ satisfies

$$
\left|\mathbf{C C}_{v}\right| \leq \max _{u \in V \cap \mathcal{L}_{i}}\left|C_{u}\right| \max _{k \leq k_{\max }^{i}}\left|V \cap \mathcal{L}_{i} \cap \mathcal{B}_{i, k}\right| \leq 2 K e^{\frac{t_{0}+t_{i}}{2}} e^{\frac{3}{2} t_{0}}=2 K e^{2 t_{0}+\frac{1}{2} t_{i}},
$$

and the lemma follows by choosing $K_{2}=2 K$.

Proof of Theorem 1.1. According to Lemma 4.3, there is a constant $K_{2}>0$ such that, a.a.s.

$$
\max _{v \in V}|\operatorname{Conn}(v)| \leq \max _{v \in V}\left|\mathbf{C C}_{v}\right| \leq K_{2} e^{2 t_{0}+\frac{1}{2} t_{\text {max }}} \leq e^{2 t_{0}+\frac{t_{\max }+3 \log R}{2}}=e^{\frac{R}{4 \alpha}+\left(\frac{8 \alpha}{\alpha-1}+\frac{3}{2}\right) \log R} .
$$

By Lemma 4.1 we obtain the upper bound for $\left|L_{1}\right|$ in the theorem.

For the lower bound, by Lemma 2.3, for any function $\omega$ tending to infinity with $n$ arbitrarily slowly, $\mu\left(B_{O}\left(r_{\max }+\omega\right)\right) \gg 1 / n$, and hence a.a.s. we find a vertex $v$ with $t_{v} \geq t_{\max }-\omega$. In such case, the degree of $v$ is, by Lemma $2.5, \mathrm{~s}$ a.a.s. between $c_{1} e^{\left.\frac{1}{2}\left(t_{\max }-\omega\right)\right)}$ and $c_{2} e^{\left.\frac{1}{2}\left(t_{\max }-\omega\right)\right)}$ (for some constants $c_{1}<c_{2}$ ), and thus, a.a.s. of the order $n^{\frac{1}{2 \alpha}+o(\omega / n)}$. The degree of a vertex is a lower bound on the size of its component, and hence Theorem 1.1 follows.

\section{References}

[ABF17] M. A. Abdullah, M. Bode, and N. Fountoulakis. Typical distances in a geometric model for complex networks. Internet Mathematics, 1, 2017. MR-3708706

[BFFK20] T. Bläsius, P. Fischbeck, T. Friedrich, and M. Katzmann. Solving vertex cover in polynomial time on hyperbolic random graphs. STACS, pages 25:1-25:14, 2020.

[BFK16] T. Bläsius, T. Friedrich, and A. Krohmer. Hyperbolic random graphs: Separators and treewidth. 24th European Symposium on Algorithms (ESA 2016), pages 15:1-15:16, 2016. MR-3550078

[BFK18] T. Bläsius, T. Friedrich, and A. Krohmer. Cliques in hyperbolic random graphs. Algorithmica, 80(8):2324-2344, 2018. MR-3800263

[BFM15] M. Bode, N. Fountoulakis, and T. Müller. On the largest component of a hyperbolic model of complex networks. Electronic J. of Combinatorics, 22(3):P3.24, 2015. MR-3386525 
[BFM16] M. Bode, N. Fountoulakis, and T. Müller. The probability of connectivity in a hyperbolic model of complex networks. Random Structures \& Algorithms, 49(1):6594, 2016. MR-3521274

[BKL17a] K. Bringmann, R. Keusch, and J. Lengler. Sampling geometric inhomogeneous random graphs in linear time. 25th European Symposium on Algorithms (ESA 2017), pages 20:1-20:15, 2017. MR-3710917

$\left[\mathrm{BKL}^{+} 17 \mathrm{~b}\right] \quad$ K. Bringmann, R. Keusch, J. Lengler, Y. Maus, and A.R. Molla. Greedy routing and the algorithmic small-world phenomenon. In Proceedings of the ACM Symposium on Principles of Distributed Computing, PODC'17, pages 371-380, New York, NY, USA, 2017. ACM.

[BKL19] K. Bringmann, R. Keusch, and J. Lengler. Geometric inhomogeneous random graphs. Theoretical Computer Science, (760):35-54, 2019. MR-3913223

[BLM13] S. Boucheron, G. Lugosi, and P. Massart. Concentration inequalities. Oxford University Press, Oxford, 2013. A nonasymptotic theory of independence, With a foreword by Michel Ledoux. MR-3185193

[BnPK10] M. Boguná, F. Papadopoulos, and D. Krioukov. Sustaining the internet with hyperbolic mapping. Nature Communications, 1:62, 2010.

[CF16] E. Candellero and N. Fountoulakis. Clustering and the hyperbolic geometry of complex networks. Internet Mathematics, 12(1-2):2-53, 2016. MR-3474052

[FK18] T. Friedrich and A. Krohmer. On the diameter of hyperbolic random graphs. volume 32, pages 1314-1334, 2018. MR-3813231

[FM18] N. Fountoulakis and T. Müller. Law of large numbers for the largest component in a hyperbolic model of complex networks. Annals of Applied Probability, 28:607-650, 2018. MR-3770885

[FvdHMS21] N. Fountoulakis, P. van der Hoorn, T. Müller, and M. Schepers. Clustering in a hyperbolic model of complex networks. Electron. J. Probab., 26(13):1-132, 2021.

[GPP12] L. Gugelmann, K. Panagiotou, and U. Peter. Random hyperbolic graphs: Degree sequence and clustering. In Automata, Languages, and Programming - 39th International Colloquium - ICALP Part II, volume 7392 of LNCS, pages 573-585. Springer, 2012.

[KL20] J. Komjathy and B. Lodewijks. Explosion in weighted hyperbolic random graphs and geometric inhomogeneous random graphs. Stochastic Processes and its Applications, 130(3):1309-1367, 2020. MR-4058275

[KM15] M. Kiwi and D. Mitsche. A bound for the diameter of random hyperbolic graphs. In Proceedings of the 12th Workshop on Analytic Algorithmics and Combinatorics ANALCO, pages 26-39. SIAM, 2015. MR-3448626

[KM18] M. Kiwi and D. Mitsche. Spectral gap of random hyperbolic graphs and related parameters. Annals of Applied Probability, 28:941-989, 2018. MR-3784493

[KM19] M. Kiwi and D. Mitsche. On the second largest component of random hyperbolic graphs. SIAM Journal on Discrete Mathematics, 33(4):2200-2217, 2019. MR-4032854

$\left[\mathrm{KPK}^{+} 10\right] \quad$ D. Krioukov, F. Papadopoulos, M. Kitsak, A. Vahdat, and M. Boguñá. Hyperbolic geometry of complex networks. Phyical Review E, 82(3):036106, 2010. MR-2787998

[MS19] T. Müller and M. Staps. The diameter of KPKVB random graphs. Advances of Applied Probability, 51(2):358-377, 2019. MR-3989518

[TM67] J. Travers and S. Milgram. The small world problem. Psychology Today, 1(1):61-67, 1967.

Acknowledgments. The authors would like to thank Antoine Barrier for providing Figure 1 and the anonymous referees for their many valuable comments which helped to significantly improve the clarity of the paper. 